\title{
Modelling Complete Power Outage Data Using Reliability
}

\author{
N. P. Akpan ${ }^{1 *}$, N. A. Bassey² \\ ${ }^{1}$ Department of Mathematics and Statistics, University of Port Harcourt, Port Harcourt, Nigeria \\ ${ }^{2}$ Department of Statistics, Akwa Ibom State College of Science and Technology, Nung Ukim, Nigeria \\ Email: nsipaulakpan@gmail.com
}

How to cite this paper: Akpan, N.P. and Bassey, N.A. (2021) Paper Title. American Journal of Operations Research, 11, 87-99. https://doi.org/10.4236/ajor.2021.112005

Received: November 27, 2020

Accepted: March 5, 2021

Published: March 8, 2021

Copyright (c) 2021 by author(s) and Scientific Research Publishing Inc. This work is licensed under the Creative Commons Attribution International License (CC BY 4.0).

http://creativecommons.org/licenses/by/4.0/

\begin{abstract}
Data on time between complete power outages, Time between Failure (TBF) in Uyo were considered. Trend test and serial correlation test were conducted graphically for the data. The tests proved that the data were identically and independently distributed (iid). Summary statistics of the data showed that complete power outage occurred 416 times between the year 2014 and 2018 . The maximum likelihood estimation method was used to estimate the parameters of Weibull 2-parameter, Normal, Lognormal 2-parameter and exponential distributions. The values of Kolmogorov-Smirnov, Anderson Darling and Chi-Square statistics were used to determine the best fit distributions. A model for the computation of reliability of electric power was then proposed.
\end{abstract}

\section{Keywords}

Reliability, Power Outage, Time between Failure, Weibull 2-Parameter Distribution

\section{Introduction}

Epileptic nature of power has been considered as a major problem to the development of Nigeria. It affects so many sectors such as educational sector, tourism, and manufacturing sector just to mention but few. It has imposed a huge cost on the affected sectors thereby leading to increased business uncertainty and lower returns on investments. Power outage has dwindled and undermined the prospect and the attractiveness of Nigeria's economy to the world and other external investors.

Nevertheless, the importance of electricity cannot be over emphasized. It plays vital role in the economic growth of both the developed and developing countries of the world. Due to its importance, household, firms, educational institu- 
tion, religion organizations, health institutions, tourists centres and research centres have taken various steps to ensure that there is steady electric power in their domains by using private fuel generators or solar generators. Also, in the manufacturing sector, Nnanna and Uzorh [1] identified power outage as the major constraint affecting the growth of manufacturing sector noting that it has hindered Nigeria's growth potential and the attractiveness of the economy to external investors. Nigeria as a nation is putting in her resources to ensure that there is no epileptic power supply in the country [1]. As Nigeria planned to be among the top 20 world economies, she must ensure that epileptic power supply and power outage are things of the past. In order to solve the problem of power outage, an in-depth study and analysis of power outages should be taken as a priority.

Reliability is a very important performance metric in system analysis, it is considered to be a good starting point for system improvements [2]. Kececioglu [3] emphasized on the importance of reliability programs stating that in the near future, only companies with knowledge and ability to control the reliability of their products will remain relevant; hence for a company to be successful in today's highly competitive and technologically complex environment, it is imperative that such company knows the reliability of its product and is able to control it. Various industries have recently increased its requirements, combined with the rapid rise in scientific and technological systems, and increased competitions of service providers to implement adequate and acceptable management strategies for the systems to enhance their availability and to comply with required standards [4]. One of the important points in this regard is that a system or service cannot be well improved upon if the knowledge about the dependability and integrity of the system is not acquired. Therefore, the knowledge of reliability is necessary for improvement in the availability of electric power. Furthermore, Goets and Villa [5] in considering the reliability of a product noted that credible measures of a product include its quality, performance, service and how cost effective it is.

Over the years, the concept of reliability has been applied to the power and manufacturing sector. Kolawole et al. [6] used the concept of reliability to obtain the reliability and performance analysis of a power generating plant in Nigeria. Adamu et al. [7] adopted the Frequency and Duration of outages (F\&D) approach of reliability to evaluate the reliability of Kainji power station in Nigeria. Dewangan et al. [8] employed the failure mode and effects analysis to investigate the reliability of turbines used in a steam power plant. Barabady [9] also used the reliability approach to determine the reliability of crushing plants of Jajarm bauxite mine in Iran. Reliasoft's Weibull ++6 software [10] was then used to estimate the parameters of the probability distributions of Weibull, Exponential and Lognormal distributions used.

Hence this study seeks to analyse the performance of power outage using the method of reliability. The rest of the paper is arranged as follows; Section 2 dis- 
cusses the materials and methods, Section 3 presents the results and discussion of the analyses and Section 4 concludes the paper.

\section{Materials and Methods}

Data for this work is a secondary data and was obtained from the record unit of the Port Harcourt Distribution Company (PhED) Uyo. The data set consisted of the up-time and down-time of electric power in Uyo Local Government area in Akwa Ibom State between January 2014 and December 2018.

\section{Weibull 2-Parameter Distribution:}

The pdf for Weibull 2-parameter distribution is given by

$$
f(t)=\frac{\alpha}{\beta}\left(\frac{t}{\beta}\right)^{\alpha-1} \mathrm{e}^{-\left(\frac{t}{\beta}\right)^{\alpha}}
$$

where $t$ is the time parameter, $\beta$ is the scale parameter and $\alpha$ is the shape parameter.

\section{Lognormal 2-Parameter Distribution:}

The pdf for lognormal 2-parameter distribution is given by

$$
f(t)=\frac{1}{t \sigma \sqrt{2 \pi}} \mathrm{e}^{\frac{-(\ln (t)-\mu)^{2}}{2 \sigma^{2}}}
$$

where $\mu$ is the mean of the natural logarithm of time between failure (TBF), $\sigma$ is the standard deviation of the natural logarithm of TBF.

\section{Normal Distribution:}

The pdf of normal distribution is given by

$$
f(t)=\frac{1}{\sigma \sqrt{2 \pi}} \mathrm{e}^{\frac{-(t-\mu)^{2}}{2 \sigma^{2}}}
$$

where $\mu$ is the mean time between failure (TBF) and $\sigma$ is the standard deviation of TBF.

\section{Exponential Distribution:}

The pdf of the exponential distribution is given by

$$
f(t)=\lambda \mathrm{e}^{-\lambda(t)}
$$

The exponential distribution plays a critical role in the study of reliability engineering due to the fact that it has a constant failure rate of $\lambda$. The distribution is also found to be very useful in modelling the lifespan of any system with mechanical and electrical components.

\subsection{Reliability}

Reliability is the probability that a product/equipment (i.e. components, system or subsystem) or process functions accurately for a given amount of time under stated condition of use without failure [11]. The reliability of a product is a function of time $(t)$ which is expressed in terms of the probability that the time to failure $(T)$ is longer than the operating time $(t)$. Thus, it suggests that reliability 
is the probability that failure has not occurred at time $(t)$, and is given by

$$
R(t)=p(T>t)
$$

where $R(0)=1$ and $R(t) \geq 0$ [12].

The cdf for reliability is denoted by $F(t)$, and for the fact that the area under the pdf is always 1, the reliability function is expressed as

$$
R(t)=p(T>t)=1-f(t)
$$

The relationship between the $\mathrm{cdf}$ and the pdf is given as

$$
F(t)=\int_{0}^{t} f(t) \mathrm{d} t
$$

Then

$$
\begin{aligned}
R(t) & =1-\int_{0}^{t} f(t) \mathrm{d} t \\
= & \int_{t}^{\infty} f(t) \mathrm{d} t
\end{aligned}
$$

where $f(t)$ is pdf of time to failure.

However, the unreliability is the same as cdf and can be seen as the probability that failure has occurred.

\subsection{Identically and Independently Distribution (iid) Assumption}

It is assumed that when data sets are iid it means that the chosen probability distribution is appropriate to model the system. If in a case that the data set does not satisfy the iid requirements and probability distributions were used in the modelling, then the outcome and/or conclusions of such analysis can be misleading [13]. For the purpose of this work, the iid assumption will be verified graphically using the trend and serial correlation tests.

\subsubsection{The Trend Test}

The trend test is usually applied in finding out the trends in the failure patterns of a machine or system. The test involves plotting the cumulative failure or the repair number against the Cumulative Time between Failures (CTBF) or Time to Repair (TTR). The trend test can be presented graphically so as to check for presence of a trend in the data set or identify whether the failure rate for individual sub-system has been increasing, decreasing or constant. The shape of the trend plot will reveal if a system is experiencing a decreasing failure rate (improving), an increasing failure rate (deteriorating) or constant (straight line). In the case of a straight line, the data set is free from any trends and is said to be identically distributed (Kumar et al., [14]; Rajaprasad, [15]; Balaraju, et al., [16]).

\subsubsection{The Serial Correlation Test}

The serial correlation test is carried out to check the relationship between two variables (ith TBF and (i-1)th TBF). In this case, the (i-1)th TBF is plotted against the ith TBF. If the resultant data points are scattered randomly and are void of a noticeable pattern, it indicates that the data set is free from serial cor- 
relation, and further suggests that the data set are independent of each other (Kumar et al., [14]; Rajaprasad, [15]; Balaraju, et al., [16]).

\subsection{Models for Data Analysis}

There are various models for analyzing the available data set. These are discussed briefly below. For the purpose of the study the system was modelled using TBF data analysis type. The goodness of-fit test is used to identify the best-fit probability distributions, while the maximum likelihood estimation method was used to estimate the parameters for the best fit distribution.

\section{TBF Data Analysis}

The TBF Data analysis deals with modelling both the times as it takes from a performed repair action to the next system failure and the time it takes to restore the system to its optimum operating state. The main focus of this method is to model the failure and repair pattern of the system. It involves fitting a probability distribution that best characterize the failure data, and also fitting a distribution that best characterize the repair data, and further estimating the parameters to fit the distributions to the different data sets [15]. The probability distributions that are commonly used for life distributions are Exponential distribution, Normal distribution, lognormal distribution and the Weibull distribution.

\subsection{Goodness-Of-Fit Test}

This test is used to identify the suitability of a given probability distribution function to interpreting the given data set. In selecting a suitable probability distribution function, is it necessary that the goodness-of-fit of the function is identified by the appropriate test. Consequently, the general principle involved in the goodness-of-fit test is to see how well the chosen distribution matches with the actual data set. Most frequently used tests are p-value test, Chi-squared test, Anderson-Darling test and Kolmogorov-Smirnov (K-S) test. The Kolmogorov-Smirnov test is mostly used for Reliability analysis (Rigdon and Basu., [17]; Rajaprasad, [15]).

\subsubsection{Modified Kolmogorov-Smirnov (K-S) Test}

Suppose that $F(t)$ is a continuous distribution to be tested as the parent distribution of a given random sample $t_{1}, t_{2}, \cdots, t_{n}$. Let $t_{(1)}, t_{(2)}, \cdots, t_{(n)}$ be the order statistics $(i=1, \cdots, n)$ and consider the largest difference at the points where empirical distribution function EDF is greater than $F(t)$, and the largest difference at the points where the EDF is smaller than $F(t)$ as

$$
\begin{aligned}
D_{k-s}^{+} & =\max _{i=1, \cdots, n}\left\{\frac{i}{n}-F\left(t_{(i)}\right)\right\} \\
D_{k-s}^{-} & =\max _{i=1, \cdots, n}\left\{F\left(t_{(i)}\right)-\frac{i-1}{n}\right\}
\end{aligned}
$$

Then the K-S Statistic is given as 


$$
D_{k-s}=\max \left\{D_{k-s}^{+}, D_{k-s}^{-}\right\}
$$

Using the K-S Statistic, the probability distribution which has the least K-S value is considered to give the best fit (Reliasoft [10], Mehrannia and Palegohar, [18]).

\subsubsection{Anderson-Darling Test}

Anderson-Darling Statistic is one of the statistics based on empirical distribution function, (EDF) which is denoted by $F_{n}(t)$ and defined as;

$$
F_{n}(t)= \begin{cases}0 & \text { if } t<T_{(1)} \\ \frac{i}{n} & \text { if } T_{i} \leq t<T_{(i+1)}, i=1, \cdots, n-1 \\ 1 & \text { if } t \geq T_{(n)}\end{cases}
$$

The plot of $F_{n}(t)$ against $t$ is a step function which gives the proportion of observation less than or equal to $t$. if $H_{O}$ is true, the EDF should mirror the null distribution $F(t ; \theta)$. The EDF statistics are based on

$$
F_{n}(t)-F(t ; \theta)
$$

Anderson-Darling Statistic is defined by

$$
A^{2}=n \int_{-\infty}^{\infty}\left[F_{n}(t)-F(t ; \theta)\right]^{2} \varphi(t) \mathrm{d} F(t ; \theta)
$$

where $\varphi(t)=[F(t ; \theta)(1-F(t ; \theta))]^{-1}$

In practice, the probability integral transform (PIT), $z_{i}=F\left(t_{i} ; \theta\right)$ is carried out, which if the parameter $\theta$ is known can produce a $z$-set which is uniformly distributed on $[0,1]$ interval. The computing formula of the statistic based on the $z$-set is as follows;

$$
A^{2}=-n-1 / n\left\{\sum(2 i-1)\left[\ln z_{(i)}+\ln \left(1-z_{(n+1-i)}\right)\right]\right\}
$$

A distribution with the least value of Anderson-Darling Statistic is considered to give the best fit (Reliasoft, [10], Mehrannia and Pakgohar, [18]).

\subsubsection{Chi-Square Test}

The Chi-Square statistic is given by

$$
x^{2}=\sum_{i=1}^{k}\left[\frac{\left(O_{i}-E_{i}\right)^{2}}{E_{i}}\right]
$$

where $K=$ number of classes or bars

$O_{i}=$ observed number of failures or repairs in the $i$ th class

$E_{i}=n P_{i}=$ expected number of failures in the ith class

$n=$ total number at risk or sample size.

$P_{i}=$ probability of a failure occurring in the $i$ th class if $H_{O}$ is true

$=F\left(t_{i}\right)-F\left(t_{i-1}\right)$

$=R\left(t_{i-1}\right)-R\left(t_{i}\right)$ for fitting failure data.

The Statistic $\left(x^{2}\right)$ has a Chi-Square distribution whose degrees of freedom is 
$K$-1-Number of estimated parameters. When using the Chi-Square Statistics, the probability distribution which gives the least $x^{2}$ value is considered to give the best fit [17]. For the purpose of the research, the modified K-S test, the Anderson-Darling test and the Chi-Square test are used.

\subsection{Parameter Estimation}

In this research, the maximum Likelihood Estimation (MLE) method shall be used for the estimation of the parameters because it is more robust and possesses the properties of unbiasness, consistency, sufficiency, and minimum variance for large samples.

\section{Maximum Likelihood Estimator (MLE)}

Generally, in finding the MLE for any probability distribution with complete data, the maximum of the following likelihood function with respect to the unknown parameters $\theta_{1}, \theta_{2}, \cdots, \theta_{k}$ must be found:

$$
L\left(\theta_{1}, \theta_{2}, \cdots, \theta_{k}\right)=\prod_{i=1}^{n} f\left(t_{i} \mid \theta_{1}, \theta_{2}, \cdots, \theta_{k}\right)
$$

This is aimed at finding the values of the estimations of $\theta_{1}, \theta_{2}, \cdots, \theta_{k}$ that render the likelihood function as large as possible for given values of $t_{1}, t_{2}, \cdots, t_{n}$.

The necessary conditions for finding the MLEs are obtained by setting to zero, the first partial derivatives of the logarithm of the likelihood function with respect to $\theta_{1}, \theta_{2}, \cdots, \theta_{k}$.

i.e.

$$
\frac{\partial \ln L\left(\theta_{1}, \theta_{2}, \cdots, \theta_{k}\right)}{\partial \theta_{i}}=0, \quad i=1,2, \cdots, k
$$

Moreover, the MLEs for some of the probability distributions are given as follows:

1) The Exponential MLE

For complete data, the MLE for the parameter $\lambda$ is given by

$$
\hat{\lambda}=\frac{n}{T}=\frac{1}{\mu}
$$

where $n=$ the number of failures

$$
T=\sum_{i=1}^{n} t_{i}
$$

2) The Normal MLE

The MLE for the population mean and population variance are given by

$$
\begin{gathered}
\hat{\mu}=\bar{t} \\
\hat{\sigma}^{2}=\frac{(n-1) S^{2}}{n}
\end{gathered}
$$

3) The Lognormal MLE

The MLE for the lognormal parameters are given by

$$
\hat{\mu}=\sum_{i=1}^{n} \frac{\ln t_{i}}{n}
$$


and

$$
\hat{S}=\sqrt{\frac{\sum_{i=1}^{n}\left(\ln t_{i}-\hat{\mu}\right)^{2}}{n}}
$$

4) The Weibull MLE

The maximum likelihood method alone cannot be used to get the parameter estimates for Weibull distribution; therefore, the method can be used jointly with Newton Raphson method. [19]. The estimates can be obtained by solving the equations;

$$
\begin{gathered}
\alpha=\left(\frac{\sum t_{i}^{\alpha} \ln t_{i}}{\sum t_{i}^{\alpha}}-\frac{1}{n} \sum \ln t_{i}\right)^{-1} \\
\beta=\left(\frac{1}{n} \sum t_{i}^{\alpha}\right)^{1 / \alpha}
\end{gathered}
$$

However, in this work, the reliability software Easy-fit was used to carry out the MLE parameter estimation and the goodness-of-fit test.

\subsection{Reliability Models}

When calculating reliabilities, the reliability function $R(t)$ is used, while cumulative distribution function, $(\mathrm{CDF}), F(t)$ is used when calculating failure probabilities. Graphically the probability density function (PDF), $f(t)$ provides a visual illustration of the failure distribution.

The probability of a failure occurring within some interval of time [a, b] may be found using any of the three probability functions, since;

$$
\operatorname{Pr}(a \leq T \leq b)=F(b)-F(a)=R(a)-R(b)=\int_{a}^{b} f(t) \mathrm{d} t
$$

The failure rate or hazard rate function which provides an instantaneous (at time $t$ ) rate of failure can be obtained as follows;

$$
\operatorname{Pr}(t \leq T \leq t+\Delta t)=R(t)-R(t+\Delta t)
$$

And the conditional probability of a failure in the time interval from $t$ to $t+$ $\Delta t$ given that it has survive to time $t$ is

$$
\operatorname{Pr}(t \leq T \leq t+\Delta t / T \geq t)=\frac{R(t)-R(t+\Delta t)}{R(t)}
$$

Then $\frac{R(t)-R(t+\Delta t)}{R(t) \Delta t}$ is the conditional probability of failure per unit of time (failure rate).

Let

$$
H(t)=\lim _{\Delta t \rightarrow 0} \frac{-(R(t+\Delta t))-R(t)}{\Delta t} \cdot \frac{1}{R(t)}=\frac{-\mathrm{d} R(t)}{\mathrm{d} t} \cdot \frac{1}{R(t)}
$$

Since $f(t)=\frac{-\mathrm{d} R(t)}{\mathrm{d} t}$ 
Therefore, Hazard Function,

$$
H(t)=\frac{f(t)}{R(t)}
$$

The Weibull failure distribution

$$
\begin{gathered}
H(t)=\frac{\alpha}{\beta}\left(\frac{t}{\beta}\right)^{\alpha-1}, \beta>0, \alpha>0, t \geq 0 \\
R(t)=\exp \left[-\int_{0}^{t} \frac{\alpha}{\beta}\left(\frac{t}{\beta}\right)^{\alpha-1} \mathrm{~d} t\right]=\exp \left(-\frac{t}{\beta}\right)^{\alpha}
\end{gathered}
$$

and

$$
f(t)=\frac{\mathrm{d} R(t)}{\mathrm{d} t}=\frac{\alpha}{\beta}\left(\frac{t}{\beta}\right)^{\alpha-1} \mathrm{e}^{-\left(\frac{t}{\beta}\right)^{\alpha}}
$$

However, if $\alpha>1$, then the hazard function is said to increase with time. If $\alpha=1$, then the hazard function remains constant. If $\alpha<1$, then the hazard function decreases over time [20].

\section{Results and Discussion}

In order to obtain the mean time between failures (MTBF) the summary statistic is needed. This is shown in Table 1.

\subsection{Mean Time Analysis}

Table 1 shows the summary statistics for the TBF data

$$
\text { MTBF }=\frac{\text { CTBF }}{\text { Total number of failures }}=\frac{43697.86}{416}=105.0429 \text { hours }
$$

From our results the Mean Time between Failures (MTBF) was found to be 105.0429 hours, this index reveals that power outage occurs after every 105.0429

Table 1. Summary statistics.

\begin{tabular}{cc}
\hline Statistic & TBF \\
Mean & 105.0429327 \\
Standard Error & 2.353005576 \\
Median & 102.785 \\
Mode & 95 \\
Standard Deviation & 47.99208539 \\
Sample Variance & 2303.24026 \\
Range & 229.58 \\
Minimum & 8.33 \\
Maximum & 237.91 \\
Sum & $43,697.86$ \\
Count & 416 \\
\hline
\end{tabular}


hours, leaving electricity unavailable and generating losses to the users. Moreover, it was observed that complete power outage occurred 416 times in Uyo L.G.A between January 2014 and December 2018.

The trend test for this work has been carried out graphically. The trend test for TBF data is shown in Figure 1.

A serial correlation test was performed to check the relationship between the two variables $\left(\mathrm{TBF}_{(\mathrm{i})}\right.$ and $\left.\mathrm{TBF}_{(\mathrm{i}-1)}\right)$. The test was done graphically as shown in Figure 2.

\subsection{Serial Correlation and Trend Tests for TBF}

As earlier stated, the trend test for this research was carried out graphically. Before the data is fitted, it is necessary to find out if the data contains any characteristics of a trend (that is if the rate of failure for the system is increasing, decreasing or remains constant). To achieve this, the cumulative time between failure and number of failure was plotted.

Figure 2 represent the scatter plots for TBF data. The scatter plot between $\left(\mathrm{TBF}_{(\mathrm{i})}\right.$ and $\left.\mathrm{TBF}_{(\mathrm{i}-1)}\right)$ reveals that the data were scattered. This proves no serial correlation between two consecutive failures which validates the assumption that TBF is independently and identically distributed.

The Easy-Fit reliability software package was used to perform the maximum likelihood estimation and the goodness-of-fit test. The results are shown in Table 2 .

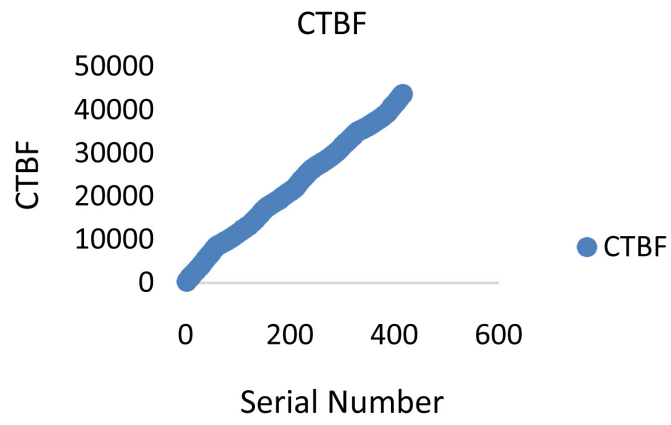

Figure 1. Trend test of CTBF.

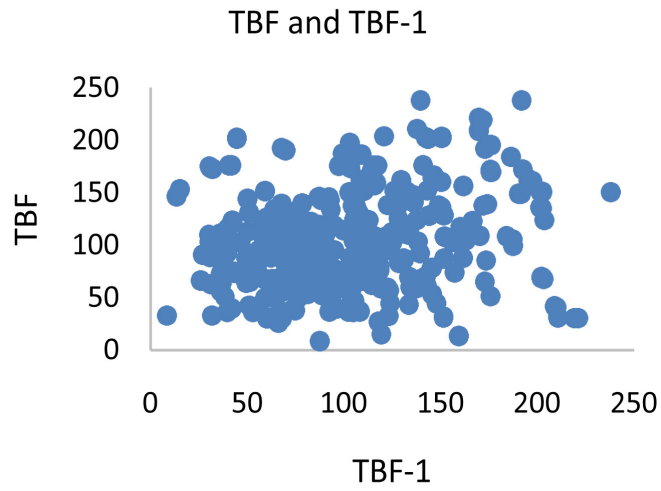

Figure 2. Correlation test for TBF. 
Table 2. Parameters estimates and goodness of fit statistics of fitted distributions for TBF data.

\begin{tabular}{|c|c|c|c|c|c|}
\hline Data & K-S & $\begin{array}{c}\text { Anderson } \\
\text { Darling }\end{array}$ & $\begin{array}{c}\text { Chi } \\
\text { Square }\end{array}$ & Best Fit & Parameter \\
\hline \multirow[t]{4}{*}{$\mathrm{TBF}$} & 0.2618 & 0.8622 & 2.8047 & Weibull & $\begin{array}{c}\alpha=2.3812 \\
\beta=118.0\end{array}$ \\
\hline & 0.0644 & 2.5285 & 27.6285 & Normal & $\begin{array}{l}\sigma=47.992 \\
\mu=105.04\end{array}$ \\
\hline & 0.2618 & 53.28 & 234.2 & Exponential & $\lambda=0.00952$ \\
\hline & 0.0780 & 3.44 & 27.274 & Lognormal & $\begin{array}{c}\sigma=0.52578 \\
\mu=4.5329\end{array}$ \\
\hline
\end{tabular}

\subsection{Maximum Likelihood Estimates and Goodness of Fit}

Table 2 shows the maximum likelihood estimates for the parameters of the four probability distributions using the TBF data, it is clear from the table that the shape parameter $(\alpha)$, and the scale parameters $(\beta)$ of Weibull 2-distribution functions were found to be 2.3812 and 118.0 respectively. The value of the mean $(\mu)$ and standard deviation $(\sigma)$ of the normal distribution were found to be 105.04 and 47.992 respectively. The value of the estimate for the parameter $(\lambda)$ of the exponential distribution is 0.00952 . The mean $(\mu)$ and the standard deviation $(\sigma)$ of the lognormal distribution were found to be 4.5329 and 0.52578 respectively.

Table 2 also shows the values of the three statistics namely KolmogorovSmirnov (K-S), Anderson-Darling and Chi-Square used for fitting the four probability distributions. With Chi-Square test, the best fit distribution is Weibull because it gives the least value (2.8047). Similarly, the Anderson Darling Statistic (0.862182) is the least when Weibull distribution is fitted. Therefore, the Weibull distribution is proposed to be the best-fit distribution for the TBF data although the K-S statistics (0.06442) is the least when the Normal distribution is fitted.

\section{Conclusions}

Reliability analysis should be considered as a priority for the management and for utilization of electricity in Nigeria. The major goal of this research was to perform the analysis of the complete power outage data by reliability method.

Based on the results of our analysis, the reliability of electric power is said to be the probability that the power outage has not taken place at time $t$, and this is given by

$$
R(t)=\exp \left\{-\left(\frac{t}{\beta}\right)^{\alpha}\right\}
$$

where $\alpha=2.3812$ and $\beta=118.0$

Hence, we have been able to develop amodel for reliability of electric power based on the best fit probability distribution (Weibull) function for the analysis 
of the electricity power outage.

\section{Conflicts of Interest}

The authors declare no conflicts of interest regarding the publication of this paper.

\section{References}

[1] Nnanna, I. and Uzorh, A.C. (2011) The Impact of Power Outage on Nigeria Manufacturing Sector. NIIE Proceedings, Ibadan, 4-6 August 2011, 45-56.

[2] Saraswat, S. and Yadava, G.S. (2008) An Overview on Reliability, Availability, Maintainability and Supportability (RAMS) Engineering. International Journal of Quality and Reliability Management, 25, 330-344. https://doi.org/10.1108/02656710810854313

[3] Kececioglu, D. (1991) Reliability Engineering Handbook. Vol. 1, Prentice Hall Inc., Englewood Cliffs.

[4] Aly, M.F., Ajety, I.H., Abdel-Magied, R.K. and Elhahion, E.K. (2018) A Comprehensive Model of Reliability, Availability and Maintainability (RAM) for Industrial Systems Evaluations. JJMIE, 12, 59-67.

[5] Goets, W. and Villa, S.D. (1998) Reliability Improvement through the Use of Plant Operating Data and the ORAP Database. $O \& M$ Conference on Modern Power Systems, London.

[6] Kolawole, A., Agbola, O.O., Ikubanni, P.P., Raji, O.G. and Osueke, C.O. (2019) Reliability and Power Loss Analysis: A Case Study of a Power Plant in Nigeria. Cogent Engineering, 6, Article ID: 1579426. https://doi.org/10.1080/23311916.2019.1579426

[7] Adamu, M.A., Adebayo, B.A., Bajoya, B.G., Ambafi, J.G. and Omokhafe, J.T. (2012) Reliability Evaluation of Kainji Hydro-Electric Power Station in Nigeria. Journal of Energy Technology and Policy, 2, 15-30.

[8] Dewangan, D.N., Kumar, M.J. and Banjare, Y.P. (2014) Reliability Investigation of Steam Turbine Used in Thermal Power Plant. International Journal of Innovation Research in Science, Engineering and Technology (IJIRSET), 3, 14915-14923.

[9] Barabady, J. (2005) Reliability and Maintenability Analysis of Crushing Plants in Jajarm Bauxite Mine of Iran. Annual Reliability and Maintainability Symposium, Alexandria, 24-27 January 2005, 109-115. https://doi.org/10.1109/RAMS.2005.1408347

[10] Reliasoft (2007) How Good Is Your Assumed Distribution's Fit. Reliability Basic Issue. http://www.weibull.com/hotwire/issue71/relbasics71.htm

[11] Pohl, E. (2010) System Reliability. John Willey and Sons, Hoboken. https://doi.org/10.1002/9780470926963.ch8

[12] Elsayed, E.A. (2012) Reliability Engineering. 2nd Edition, John Wiley and Sons, Hoboken.

[13] Kumar, U. and Klefsjo, B. (1992) Reliability Analysis of Hydraulic System of LHD Machine Using the Power Law Process Model. Reliability Engineering and System Safety, 35, 217-224. https://doi.org/10.1016/0951-8320(92)90080-5

[14] Kumar, U., Klefsjo, B. and Granholm, S. (1989) Reliability Investigation for a Fleet of Load Haul Dump Machines in a Swedish Mine. Reliability Engineering and System Safety, 28, 36-42. https://doi.org/10.1016/0951-8320(89)90004-5

[15] Rajaprasad, S.V.S. (2018) Investigation of Reliability, Maintainability and Availabil- 
ity of a Paper Machine in an Integrated Pulp and Paper Mill. International Journal of Engineering, Science and Technology, 10, 43-56.

https://doi.org/10.4314/ijest.v10i3.5

[16] Balaraju, J., Govinda Raj, M. and Murthy, C.H.S.N. (2018) Estimation of Reliability-Based Maintenance Time Intervals of Load-Haul-Dumper in an Underground Coal Mine. Journal of Mining \& Environment, 9, 761-770.

[17] Rigdon, S.E. and Basu, A.P. (2000) Statistical Methods for the Reliability of Repairable Systems. John Wiley \& Sons, Inc., Hoboken.

[18] Mehrannia, H. and Pakgohar, A. (2014) Using Easy Fit Software for Goodness-of-Fit Test and Data Generation. International Journal of Mathematical Archive, 5, 118-124.

[19] Dibal, N.P., Bakari, H.R. and Yahaya, A.M. (2016) Estimating the Parameters in the Two-Parameter Weibull Model Using Simulation Study and Real-Life Data. IOSR Journal of Mathematics, 12, 38-42.

[20] Collett, D. (2009) Modelling Survival Data in Medical Research. 2nd Edition, Chapman and Hall/CRC, London. 